\title{
Energy scaling, second Stokes oscillation and Raman gain-guiding in monolithic diamond Raman lasers
}

\author{
Vasili G. Savitski, Giorgos Demetriou, Sean Reilly, Hangyu Liu, Erdan Gu, Martin D. Dawson, \\ Fellow, IEEE, and Alan J. Kemp, Member, IEEE
}

\begin{abstract}
Energy scaling of the 1st Stokes oscillation is compared in micro-lensed and plane-plane monolithic diamond Raman lasers under $532 \mathrm{~nm}$ pumping. A maximum Raman pulse energy of $92 \mu \mathrm{J}$ at $573 \mathrm{~nm}$ was achieved with the micro-lensed device, while in a plane-plane configuration the maximum Raman pulse energy was $3.1 \mathrm{~mJ}$. 2nd Stokes generation at $620 \mathrm{~nm}$ in 2 and $1 \mathrm{~mm}$ long micro-lensed monolithic diamond Raman lasers is also reported. The best conversion efficiency from the pump at $532 \mathrm{~nm}$, namely $63 \%$, was observed in a $2 \mathrm{~mm}$ long crystal at the pump pulse intensity of $4.5 \mathrm{GW} / \mathrm{cm}^{2}$. By measuring the output Raman laser beam caustic it was found that the 2 nd Stokes intracavity beam radius at the output coupler of the micro-lensed device is at least two times smaller than that expected from the ABCD matrix calculations of the resonator mode. A Raman gain-guiding mechanism is suggested to explain this difference.
\end{abstract}

Index Terms - Diamond Raman lasers, Raman scattering, Solid lasers

\section{INTRODUCTION}

A range of important applications in biomedical instrumentation require laser wavelengths often unavailable from commercial systems. For example, photo-acoustic imaging $[1,2]$ is a powerful and non-invasive technique for biomedical imaging. Absorption of a laser pulse at a tissue-specific wavelength results in ultrasound emission that can be used to form a detailed and tissue-specific image [3]. The ideal laser for such application would be a tunable laser (e.g. dye-based lasers [4-6] or OPO systems [7-9]); however, these systems are often complex and expensive. Simpler, more compact and less expensive devices for frequency conversion of robust and commercially-available lasers are desirable for such applications. Solid-state Raman lasers are such a solution. A range of visible and UV wavelengths suitable for biomedical applications have been reported, in both $\mathrm{CW}$ and pulsed regimes, using stimulated Raman scattering, sometimes with a subsequent frequency doubling or sum frequency mixing step [10-15]. Wavelength conversion from green to yellow was recently demonstrated based on a compact monolithic diamond

Manuscript received, 2018. This work was supported by the European Research Council (727738); UK EPSRC (EP/P00041X/1); Fraunhofer UK Research Ltd.; Royal Academy of Engineering.

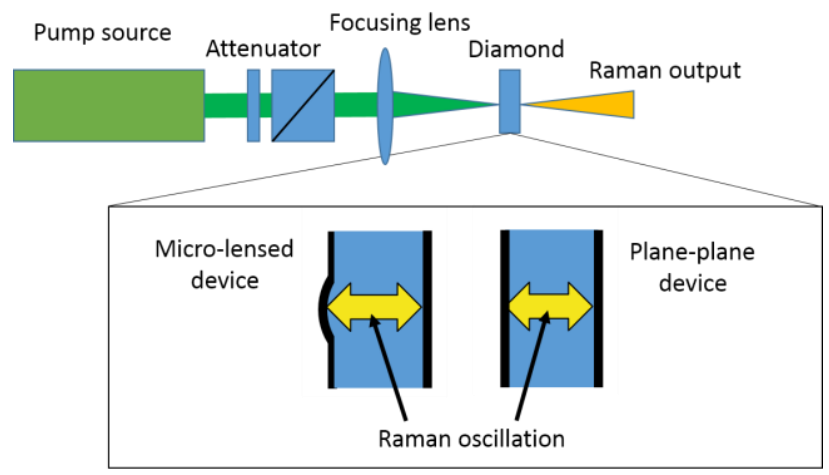

Fig. 1. Experimental setup used for energy scaling and $2^{\text {nd }}$ Stokes oscillation experiments in micro-lensed and plane-plane devices.

Raman laser (DRL) design under nano- and picosecond pumping [16-18].

In a previous proof of concept monolithic DRL demonstration [16] the Raman conversion efficiency achieved was $84 \%$ at $4.2 \mathrm{GW} / \mathrm{cm}^{2}$ of pump intensity at $532 \mathrm{~nm}$. The combined maximum Raman pulse energy was $13.4 \mu \mathrm{J}$. Scaling of the pulse energy of the Raman emission and broadening of the colour palette available with a monolithic DRL [16] were the main subjects of the present research. In this work we report an improvement of two orders of magnitude in the pulse energy from a monolithic DRL working in the yellow spectral range, and 2nd Stokes Raman oscillation at $620 \mathrm{~nm}$.

Energy scaling in micro-lensed and plane-plane monolithic devices will be compared in sub-section II,A of the "Experimental results" section. This will be followed by a description of the performance of a micro-lensed diamond Raman converter at $620 \mathrm{~nm}$ - the wavelength corresponding to the $2^{\text {nd }}$ Stokes Raman shift in diamond (sub-section $I I, B$ ). In sub-section II, $C$ the measurements of the intracavity Raman mode sizes in micro-lensed devices will be presented. In Section III we suggest and discuss a Raman gain- guiding mechanism to explain the phenomena observed in sub-section II, C.

The authors are with the Institute of Photonics, Department of Physics, SUPA, University of Strathclyde, 99 George Street, Glasgow G1 1RD, UK (+44 141 548 5736; e-mail: vasili.savitski@ strath.ac.uk). 


\section{EXPERIMENTAL RESULTS}

\section{A. $1^{\text {st }}$ Stokes emission energy scaling}

In this sub-section a side-by-side comparison of the energy scaling in micro-lensed and plane-plane monolithic DRL is presented. The experimental setup scheme is shown in Fig. 1. Both the micro-lensed and plane-plane devices were $2 \mathrm{~mm}$ thick and were fabricated from material supplied by Element Six Ltd with properties similar to those described in [16]. An array of micro-lenses was etched on the diamond surface to create a micro-lensed device (see Fig. 1), using a technique described in detail in [19]. The micro-lenses have parameters similar to the ones reported in [16]. The radius of curvature (RoC) of the micro-lenses was $13 \mathrm{~mm}$. The input mirror was deposited onto the micro-lensed surface of the diamond and on one of the two surfaces of the plane-plane diamond. For both diamonds it had reflectivity (R) of $18 \%$ at $532 \mathrm{~nm}$, was highly-reflective (HR) at $573 \mathrm{~nm}$, and had $\mathrm{R}=15 \%$ at $620 \mathrm{~nm}$. The output mirror for both diamonds was $\mathrm{HR}$ at $532 \mathrm{~nm}$, giving a double pass for the pump, and had $\mathrm{R}=50 \%$ at $573 \mathrm{~nm}$ and $\mathrm{R}=15 \%$ at $620 \mathrm{~nm}$.

The limitations on the power scaling of the monolithic device and plane-plane device are different. For the micro-lensed monolithic DRL characterised in this paper, the dominant factor controlling the transverse mode is the stable plano-concave cavity. The mode size is mainly determined by the RoC of the micro-lenses etched on one of the surfaces of the diamond (13 $\mathrm{mm}$ is the longest achievable at present). This short RoC will result in a small intracavity mode radius meaning that energy scaling of the Raman emission will be limited by the damage threshold of the coatings deposited on the diamond surfaces. For instance in a $2 \mathrm{~mm}$ long device the calculated TEM $_{00}$ Raman laser mode radius using the ABCD matrix is $24 \mu \mathrm{m}$ [16]. The pump spot radius used by us previously was $9 \mu \mathrm{m}[16]$ and the maximum achievable pulse energy of $13.4 \mu \mathrm{J}$ was limited by the damage of the coatings. Therefore the first approach for energy scaling was to increase the pump spot radius. For the work under discussion in this paper, a more powerful pump laser (Elforlight FQS laser) was used and the pump radius was increased to $23 \mu \mathrm{m}$. This allowed the Raman output energy to be scaled up as the onset of coating damage happening at higher pulse energies due to the larger pump spot size. This pump laser emitted near diffraction limited pulses of $5 \mathrm{~ns}$ duration at a maximum energy of $155 \mu \mathrm{J}$.

In the case of the plane-plane device, where the Raman laser mode size is less constrained, energy scaling can be achieved by increasing the pump spot radius to values much larger than $23 \mu \mathrm{m}$. Therefore in the $2^{\text {nd }}$ approach, pump spot radii of 30,80 and $195 \mu \mathrm{m}$, along with a high pulse energy Continuum Minilite II laser (pulse duration of $5 \mathrm{~ns}$, maximum pulse energy $10 \mathrm{~mJ}$, wavelength $532 \mathrm{~nm}$ ), were used to pump the monolithic planeplane Raman cavity.

In the case of the micro-lensed device a maximum Raman pulse energy of $92 \mu \mathrm{J}$ at a repetition rate of $10 \mathrm{kHz}$ was achieved (Fig 2 (a)) with a maximum total conversion efficiency of $60 \%$ at pump intensity of $3.7 \mathrm{GW} / \mathrm{cm}^{2}$. This energy is seven times higher than the $13.4 \mu \mathrm{J}$ achieved in [16]. Approximately $60 \mu \mathrm{J}$ of this maximum energy was at the $1^{\text {st }}$ Stokes and the remaining

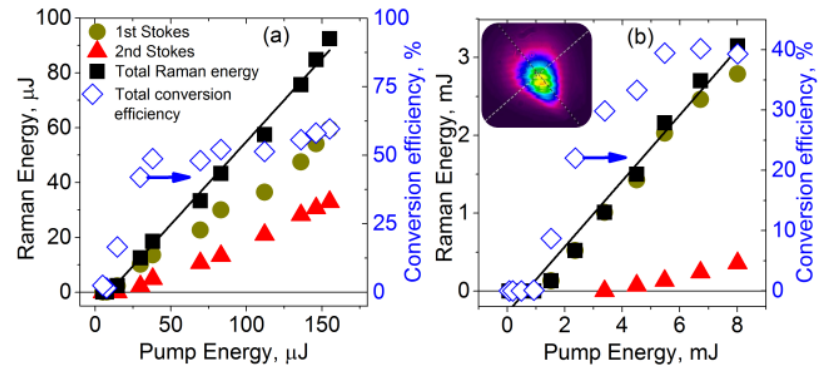

Fig. 2. Power transfer and conversion efficiency of (a) micro-lensed monolithic DRL with the pump spot radius of $23 \mu \mathrm{m}$ and (b) plane plane monolithic DRL with the pump spot radius of $195 \mu \mathrm{m}$. Inset in (b): output beam profile of the plane-plane monolithic DRL. Solid lines are the linear fits to the total Raman energy with the slopes of $61 \%$ (a) and $42 \%$ (b)

$32 \mu \mathrm{J}$ at the $2^{\text {nd }}$ Stokes (Fig. 2 (a)). The slope efficiency of the total Raman output was $61 \%$ (solid line in Fig. 2 (a)). The $1^{\text {st }}$ Stokes pulse duration was $3.5 \mathrm{~ns}$ and the $\mathrm{M}^{2}$ beam quality was measured to be 8 . The $2^{\text {nd }}$ Stokes pulse duration was $2.3 \mathrm{~ns}$; the $\mathrm{M}^{2}$ beam quality was not measured. It is possible that the $1^{\text {st }}$ Stokes beam quality was degraded by the emission of the $2^{\text {nd }}$ Stokes.

The highest pulse energy reported so far from a DRL operating in the visible spectral range [16-18, 20] is $0.3 \mathrm{~mJ}$ [20].

With a plane-plane device and a pump spot radius of $30 \mu \mathrm{m}$, coating damage was observed at pump energies of $1 \mathrm{~mJ}$. A maximum $1^{\text {st }}$ Stokes energy of only $0.8 \mathrm{~mJ}$ was measured with the pump spot radius of $80 \mu \mathrm{m}$. This $1^{\text {st }}$ Stokes energy saturated at a pump pulse energy of $4 \mathrm{~mJ}$ and was limited by the presence of the $2^{\text {nd }}$ Stokes emission, oscillating at the pump pulse energy $>0.8 \mathrm{~mJ}$. With a pump spot radius of $195 \mu \mathrm{m}$, a maximum Raman output pulse energy of $3.1 \mathrm{~mJ}$ at a repetition rate of 10 $\mathrm{Hz}$ was demonstrated, for a pump pulse energy of $8 \mathrm{~mJ}$ (Fig. 2(b)) with a maximum total conversion efficiency of $40 \%$ at pump intensity of $2.7 \mathrm{GW} / \mathrm{cm}^{2}$. This is a 300 -fold increase in Raman output pulse energy when compared with the results presented by us before [16]. As can be seen from Fig. 2 (b), almost all of this energy was contained in the $1^{\text {st }}$ Stokes emission. The slope efficiency of the total Raman output was 42\% (solid line in Fig. 2 (b)). The Raman pulse duration was 5 ns and the beam $\mathrm{M}^{2}$ factor was 10 . It should be noted that the $\mathrm{M}^{2}$ factor of the pump was 7 .

For both micro-lensed and plane-plane devices, the output power of the $2^{\text {nd }}$ Stokes was measured only in one direction, from the output mirror. However, given the equal reflectivities of the input and output mirrors at the $2^{\text {nd }}$ Stokes wavelength (15\%), one would expect the total $2^{\text {nd }}$ Stokes power to be at least two times higher. For that reason we believe the maximum total conversion efficiency (Fig. 2) for both devices is higher: at least $81 \%$ for micro-lensed device and $44 \%$ for the plane-plane device. Here, it should be mentioned that oscillation at the $1^{\text {st }}$ Stokes wavelength was primarily targeted in these experiments and therefore detailed characterization of the $2^{\text {nd }}$ Stokes emission was not undertaken.

A lack of parallelism of the surfaces of the plane-plane device (wedge angle of $0.1^{\circ}$ ) caused coma-type aberration of the output Raman beam (see inset in Fig. 2 (b)) and created additional passive losses for the Stokes emission, which limits 
the total conversion efficiency in this device in comparison with the micro-lensed one. Were diamond available with better surface parallelism, higher pulse energies and improved beam quality would be expected.

In summary, with the micro-lensed device, a seven fold increase in the output Raman pulse energy (to $92 \mu \mathrm{J}$ ) at $573 \mathrm{~nm}$ was achieved in comparison with our previous report in [16], by matching the pump spot radius to that of the expected intracavity Raman mode and using a higher energy pump laser. With the plane-plane device, a 300-fold increase in Raman output pulse energy was achieved at $573 \mathrm{~nm}$ (comparing with the results in [16]) by scaling the pump spot radius and pump pulse energy further.

\section{B. $2^{\text {nd }}$ Stokes oscillation in micro-lensed devices}

The spectral characteristics of the coatings deposited onto the surfaces of a monolithic diamond Raman laser determine how much of each Stokes component will be generated [16].

To explore the possibility of extending the spectral coverage of a monolithic diamond Raman laser to the red spectral range, corresponding to the $2^{\text {nd }}$ Stokes emission at $620 \mathrm{~nm}$ when pumped at $532 \mathrm{~nm}$, two micro-lensed diamond devices with the lengths of 2 and $1 \mathrm{~mm}$ were manufactured with a micro-lens RoC of $13 \mathrm{~mm}$ in the $2 \mathrm{~mm}$ device and an RoC of $11.5 \mathrm{~mm}$ in the $1 \mathrm{~mm}$ one. The diamond was grown by Element 6 (UK) and had an absorption coefficient of $0.05 \mathrm{~cm}^{-1}$ for the $2 \mathrm{~mm}$ crystal and $0.1 \mathrm{~cm}^{-1}$ for the $1 \mathrm{~mm}$ one (diamond heat spreader) at 532 $\mathrm{nm}$. The $2 \mathrm{~mm}$ long sample was cut for propagation along $\langle 110$, and the $1 \mathrm{~mm}$ long one was cut for propagation along

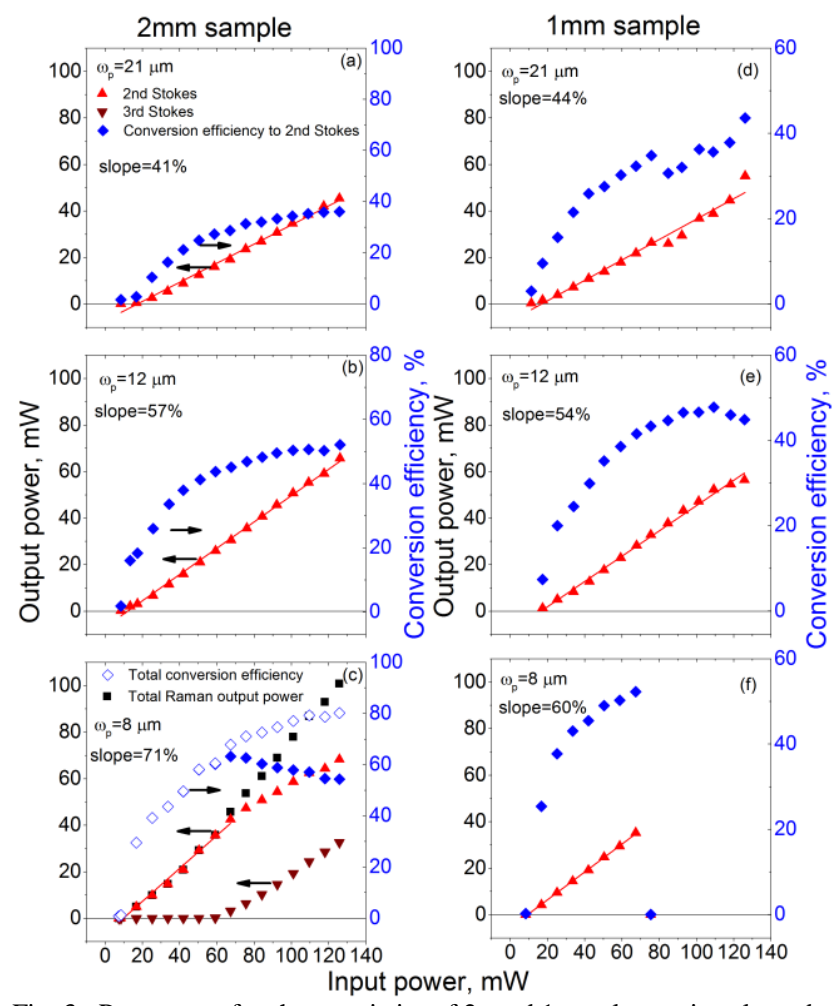

Fig. 3. Power transfer characteristics of 2- and $1 \mathrm{~mm}$ long micro-lensed Raman converters for different focusing lenses. Solid lines are the linear fits to the $2^{\text {nd }}$ Stokes output power.

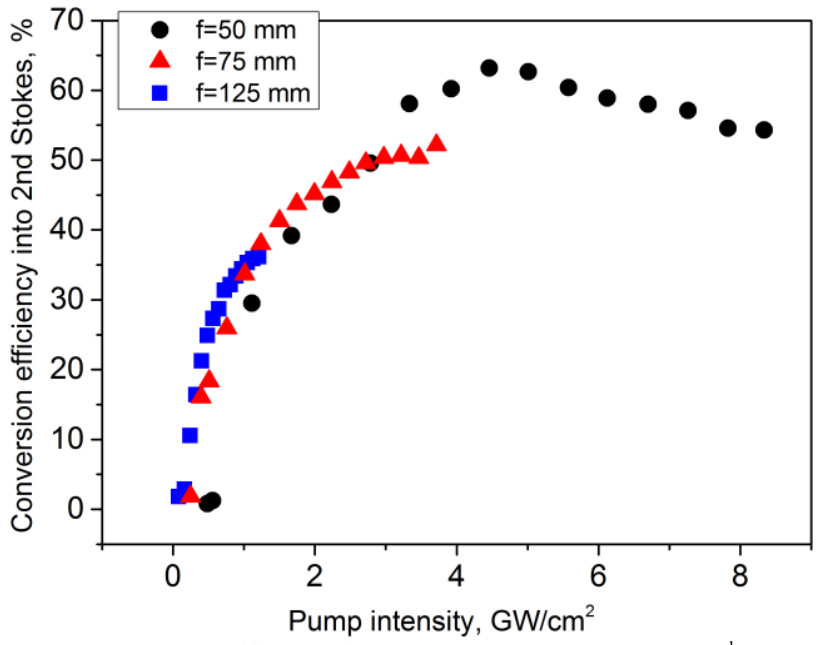

Fig. 4. Conversion efficiency from the pump at $532 \mathrm{~nm}$ to the $2^{\text {nd }}$ Stokes at $620 \mathrm{~nm}$ as a function of pump intensity.

$<100\rangle$. The pump beam was polarized along $\langle 100\rangle$ in both cases.

The coatings parameters of the input mirror (deposited onto the structured surface) were: $\mathrm{HR}$ at 573,620 and $676\left(3^{\text {rd }}\right.$ Stokes) $\mathrm{nm}$, and $\mathrm{R}=16 \%$ at $532 \mathrm{~nm}$. The output mirror was HR coated for 532 and $573 \mathrm{~nm}$, and had $\mathrm{R}=50 \%$ at $620 \mathrm{~nm}$ and $\mathrm{R}=20 \%$ at $676 \mathrm{~nm}\left(3^{\text {rd }}\right.$ Stokes $)$. An Elforlight SPOT laser with a pulse duration of $1.5 \mathrm{~ns}$ at $532 \mathrm{~nm}$ operating at a pulse repetition rate of $10 \mathrm{kHz}$ was used as a pump.

The micro-lensed converters were tested with different pump focusing lenses: 125,75 and $50 \mathrm{~mm}$, which provided pump spot radii of, correspondingly, 21, 12 and $8 \mu \mathrm{m}$. Power transfer characteristics of the devices are shown in Fig. 3. The output power of the $1^{\text {st }}$ Stokes (due to leakage from the output mirror) never exceeded $0.3 \mathrm{~mW}$, and any detectable laser output power from the $3^{\text {rd }}$ Stokes was measured only with the $8 \mu \mathrm{m}$ pump spot radius and only for the $2 \mathrm{~mm}$ long device. The highest conversion efficiency of $63 \%$ from the pump $(532 \mathrm{~nm})$ to the $2^{\text {nd }}$ Stokes $(620 \mathrm{~nm})$ was observed in the $2 \mathrm{~mm}$ long device with the pump spot radius of $8 \mu \mathrm{m}$. This efficiency was reached at a pump peak intensity of $4.5 \mathrm{GW} / \mathrm{cm}^{2}$ (Fig. 4). Higher pump intensities resulted in laser oscillation at the $3^{\text {rd }}$ Stokes wavelength (Fig. 3 (c)) therefore reducing the $2^{\text {nd }}$ Stokes conversion efficiency from then on (Fig. 4). The total Raman conversion efficiency reached $80 \%$ at a pump peak intensity of $8.3 \mathrm{GW} / \mathrm{cm}^{2}$ (the corresponding conversion efficiency to the $2^{\text {nd }}$ Stokes dropped to $54 \%$ at this intensity; see Fig. 3 (c)). The maximum $2^{\text {nd }}$ Stokes output power reached $68.3 \mathrm{~mW}$ and that of the $3^{\text {rd }}$ Stokes was $32.6 \mathrm{~mW}$ in the $2 \mathrm{~mm}$ long device for the maximum pump power of $126 \mathrm{~mW}$ with the pump spot radius of $8 \mu \mathrm{m}$. In the case of the $1 \mathrm{~mm}$ long sample, damage of the coatings with the pump spot radius of $8 \mu \mathrm{m}$ prevented measurements at pump intensities higher than $3.9 \mathrm{GW} / \mathrm{cm}^{2}$ (pump power of $67 \mathrm{~mW}$ ). By further reducing the reflectivity of the input and output mirrors at the $3^{\text {rd }}$ Stokes wavelength, it 
should be possible to reach higher conversion efficiency from the pump to the $2^{\text {nd }}$ Stokes by eliminating conversion of the $2^{\text {nd }}$ Stokes emission into the $3^{\text {rd }}$ Stokes.

The slope efficiency of the $2^{\text {nd }}$ Stokes output power (solid lines in Fig. 3) increased with decreasing pump spot size: from $41 \%$ with the $21 \mu \mathrm{m}$ pump spot radius to $71 \%$ with the $8 \mu \mathrm{m}$ pump spot radius in the $2 \mathrm{~mm}$ long device, whilst in the case of the $1 \mathrm{~mm}$ long device, the corresponding increase for the same pump spot sizes was from $44 \%$ to $60 \%$.

The $2^{\text {nd }}$ Stokes pulse duration was measured to be $1 \mathrm{~ns}$ FWHM at maximum pump power with the pump spot radii of 21 and $12 \mu \mathrm{m}$ for both devices and with the pump spot radius of $8 \mu \mathrm{m}$ for the $1 \mathrm{~mm}$ device. In the $2 \mathrm{~mm}$ device, with tighter pump focusing of $8 \mu \mathrm{m}$, the $2^{\text {nd }}$ Stokes pulse duration was 1.5 ns at maximum pump power and the $3^{\text {rd }}$ Stokes pulse duration in that case was $1.1 \mathrm{~ns}$. The $\mathrm{M}^{2}$ of the $2^{\text {nd }}$ Stokes beam was $2.0 \times 1.7$ for the $2 \mathrm{~mm}$ long device and $6 \times 4$ for the $1 \mathrm{~mm}$ long one. The reason for this difference in the beam quality is not completely clear at the moment. One of the reasons could be related to the difference in the quality of micro-lenses in two devices.

To summarize, a maximum conversion efficiency of $63 \%$ from the pump at $532 \mathrm{~nm}$ to the $2^{\text {nd }}$ Stokes at $620 \mathrm{~nm}$ was observed in a $2 \mathrm{~mm}$ long monolithic DRL at the pump pulse intensity of $4.5 \mathrm{GW} / \mathrm{cm}^{2}$. This demonstrates the flexibility of the monolithic DRL devices in extending the colour palette of commercial green laser sources.

The slope efficiency of the $2^{\text {nd }}$ Stokes oscillation (Fig. 3) increases for smaller pump spot sizes in both micro-lensed devices. It was also noted that the performance of the monolithic micro-lensed device in [16] was better with smaller pump spot radii. In a conventional laser [21], the slope efficiency would primarily be governed by the spatial overlap of the pump and laser beams, the quantum defect, and the losses. One would expect that the spatial overlap and therefore the slope efficiency will be better when the pump spot size is close to the intracavity Raman laser mode size. In our case, this overlap was expected to be highest with the $21 \mu \mathrm{m}$ pump spot radius, as the intracavity $\mathrm{TEM}_{00}$ Raman laser mode size at 620 $\mathrm{nm}$ is 24 and $20 \mu \mathrm{m}$ for 2 - and $1 \mathrm{~mm}$ long micro-lensed devices respectively, as calculated using $\mathrm{ABCD}$ matrix formalism. However, the highest slope efficiency was observed with the pump spot radius of $8 \mu \mathrm{m}$, which is $\sim 3$ times smaller than the expected, from the ABCD matrix, Raman mode size. As a first step towards explaining the observed phenomena, the intracavity $2^{\text {nd }}$ Stokes Raman mode size was measured and the results are presented in the next sub-section.

\section{Intracavity $2^{\text {nd }}$ Stokes beam size in micro-lensed devices}

The laser mode size, on the output (plane) mirror, of the $2^{\text {nd }}$ Stokes emission for both the 2- and $1 \mathrm{~mm}$ long micro-lensed converters was calculated by measuring the $2^{\text {nd }}$ Stokes output beam caustic after a lens placed in the output beam. $4 \sigma$ beam diameters were measured with a CCD camera at a pump (532 $\mathrm{nm}$ ) intensity of $I_{p} \sim 1 \mathrm{GW} / \mathrm{cm}^{2}$. The quality factor, $\mathrm{M}^{2}$, of the output beam at this pump intensity was also measured and taken into account. A fit to the data was obtained using the $\mathrm{ABCD}$

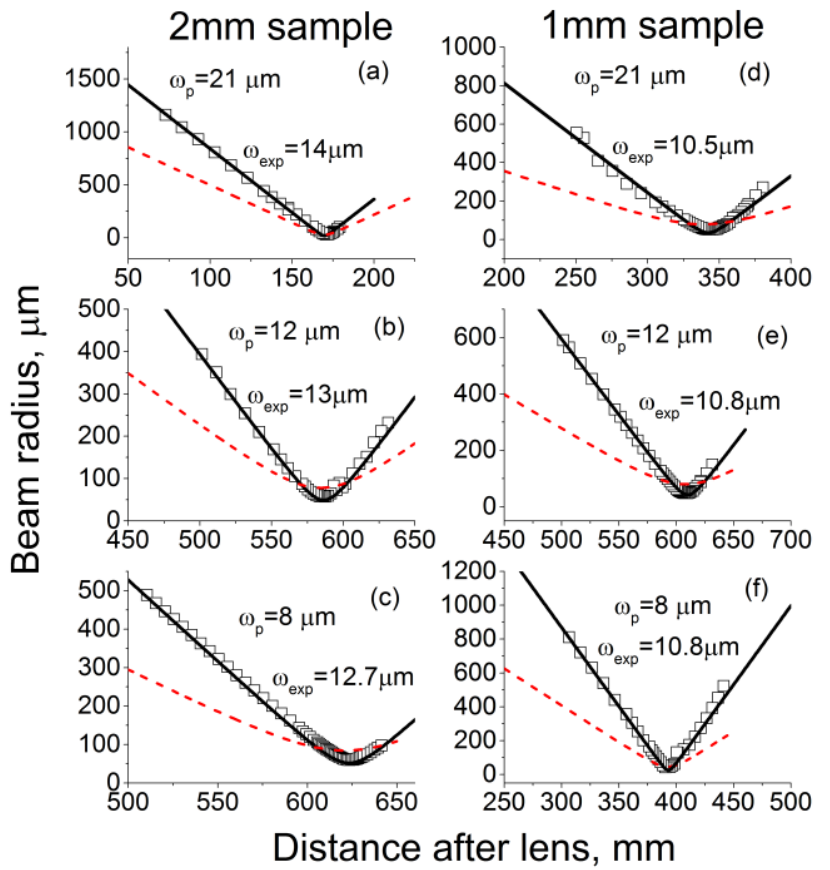

Fig. 5. Measured (squares) and simulated (solid lines) radii of the $2^{\text {nd }}$ Stokes beam caustics of the 1 - and $2 \mathrm{~mm}$ micro-lensed Raman converters for different pump spot radii $\omega_{\mathrm{p}}$. The $2^{\text {nd }}$ Stokes laser mode radii $\omega_{\exp }$ on the output coupler which were kept as free fit parameters each time are also shown. The red dashed lines are the results of simulations with the $2^{\text {nd }}$ Stokes intracavity Raman beam radii on the output coupler of 24 (for the $2 \mathrm{~mm}$ sample) and $20 \mu \mathrm{m}$ (for the $1 \mathrm{~mm}$ sample), as were expected from the calculation using the $\mathrm{ABCD}$ formalism.

matrix approach, with the $2^{\text {nd }}$ Stokes laser mode radius $\omega_{\exp }$ on the output coupler as the only fitting parameter: these are the black solid lines in Fig. 5. The results of the measurements and fit for different pump spot radii are shown in Fig. 5.

We found that for the $2 \mathrm{~mm}$ long micro-lensed device the $2^{\text {nd }}$ Stokes intracavity mode radius on the output mirror is between $14.0 \pm 0.5$ and $12.7 \pm 0.3 \mu \mathrm{m}$ for different pump spot sizes used (Fig. 5 (a)-(c)). For the $1 \mathrm{~mm}$ long device, this radius is smaller: between $10.5 \pm 0.5$ and $10.8 \pm 0.5 \mu \mathrm{m}$ (Fig. 5 (d)-(f)). These values are $\sim 2$ times smaller than the ones obtained by calculating the diamond monolithic resonator mode radius using the ABCD matrix formalism $(24 \mu \mathrm{m}$ in $2 \mathrm{~mm}$ long device and $20 \mu \mathrm{m}$ in $1 \mathrm{~mm}$ long one).

These measurements indicate that the spatial overlap between the pump and the intracavity Raman beams would be better for pump spot sizes smaller than one would expect from calculations based on the ABCD formalism. This may explain why the $2^{\text {nd }}$ Stokes slope efficiencies (Fig. 3) are higher for tighter focusing in both 1 - and $2 \mathrm{~mm}$ long micro-lensed devices.

Thermal lensing in a Raman laser could in principle be responsible for the smaller than expected $2^{\text {nd }}$ Stokes mode size on the output mirror in the micro-lensed devices. However, the focal length of such a lens would have to be $<1 \mathrm{~mm}$ in both 2and $1 \mathrm{~mm}$ devices, to have the effect observed experimentally. This is based on ABCD calculations of the Raman resonator mode size. Such a focal length would correspond to an average power for the $2^{\text {nd }}$ Stokes emission between 0.7 and $4.6 \mathrm{~kW}$ for pump spot radii between 8 and $21 \mu \mathrm{m}$ [22]. These powers are 
significantly higher (about four orders of magnitude for the 8 $\mu \mathrm{m}$ pump radius) than the average powers observed experimentally. Also, assuming a dynamic heating case in pulsed Raman lasers and using equation (2) in [23], the focal length of the thermal lens in a $2 \mathrm{~mm}$ long diamond at a $2^{\text {nd }}$ Stokes pulse energy of $7 \mu \mathrm{J}$ was calculated to be $\sim 7 \mathrm{~m}$. This is much longer than the focal length of $<1 \mathrm{~mm}$ estimated above.

In the next section we suggest another mechanism which can explain why the measured values of the intracavity Raman mode size in micro-lensed monolithic devices at $620 \mathrm{~nm}$ are $~ 2$ times smaller than the ones obtained by calculating the monolithic resonator mode radius with the $\mathrm{ABCD}$ matrix formalism.

\section{GAUSSIAN DUCT IN RAMAN LASERS}

\section{A. ABCD matrix of the Gaussian duct in the case of Raman gain-guiding}

A possible explanation of the observed difference between the measured $2^{\text {nd }}$ Stokes intracavity Raman beam size and that expected from the ABCD matrix, is based on the effect of Raman gain-guiding. This effect was predicted theoretically as early as 1975 [24], but, to the best of our knowledge, no attempt was made to prove this mechanism experimentally in Raman lasers.

Assuming Raman gain-guiding takes place, the transverse resonator mode in the Raman gain material will be modified by a Gaussian duct [21] due to transverse change of the Raman laser gain near the optical axis [21, 24-26].

First, we evaluate the ABCD matrix of this duct for the case of Raman gain-guiding. In general, the ABCD matrix of a Gaussian duct in a medium with length $L$ and refractive index $n$ is [21]:

$$
M=\left(\begin{array}{cc}
\cos (\gamma L) & \frac{\sin (\gamma L)}{n \gamma} \\
-n \gamma \sin (\gamma L) & \cos (\gamma L)
\end{array}\right)
$$

In the case of Raman gain-guiding, the parameter $\gamma$ accounts for the variation of the Raman laser gain $G$ along the transverse coordinate $\mathrm{x}$ [21]:

$$
G(x)=G_{0}-1 / 2 G_{2} x^{2}(2),
$$

and can be expressed, according to [21], as:

$$
\gamma=\frac{\sqrt{2}(i+1)}{2} \sqrt{\frac{\lambda_{s} G_{2}}{2 \pi n}}(3),
$$

where $\lambda_{s}$ is the wavelength of the Raman laser emission in vacuum, $G_{0}$ is the peak Raman gain and $G_{2}$ accounts for the Raman gain decrease with the transverse coordinate $x$.

The transverse variation of the Raman laser gain $G(x)$ can also be expressed as:

$$
G(x)=2 g I_{P} \exp \left(-\frac{2 x^{2}}{\omega_{P}^{2}}\right)
$$

where $g$ is the Raman gain coefficient, $I_{P}$ is the pump laser onaxis peak intensity, and $\omega_{P}$ is the $1 / e^{2}$ pump beam radius. The coefficient "2" before the Raman gain in (4) is added to account for backward Raman scattering. Here we ignore any thermal effects, as was previously discussed (end of Section II). In the approximation of beam propagation close to the optical axis $\left(x<<\omega_{P}\right)$, eq. (4) can be re-written as:

$$
G(x)=2 g I_{P}\left(1-\frac{2 x^{2}}{\omega_{P}^{2}}\right)=2 g I_{P}-2 g I_{P} \frac{2 x^{2}}{\omega_{P}^{2}}
$$

Comparing (2), (3) and (5), the parameter $\gamma$ can now be written as:

$$
\gamma=\frac{\sqrt{2}(i-1)}{\omega_{P}} \sqrt{\frac{\lambda_{s} g I_{P}}{\pi n}}
$$

The ABCD matrix of the Gaussian duct in the case of Raman gain-guiding can now be evaluated from (1) using the parameter $\gamma$ expressed in (6).

\section{B. Calculation of the resonator mode size in micro-lensed devices in the case of Raman gain-guiding}

The resonator mode at the output mirror of the micro-lensed device can be calculated using the $A B C D$ matrices of the resonator mirrors and Gaussian duct.

An example of this calculation for the $1^{\text {st }}$ Stokes wavelength of $573 \mathrm{~nm}$ is presented in Fig. 6. The Raman mode size was calculated as a function of the pump intensity $I_{p}$ for the pump spot radius of $\omega_{\mathrm{p}}=8 \mu \mathrm{m}$ and the Raman gain of $\mathrm{g}=4.6 \mathrm{~cm} / \mathrm{GW}$ (the reason for this choice of the Raman gain will be explained below). The calculations are done for a $2 \mathrm{~mm}$ long micro-lensed device (solid line) and for a plane-plane device of the same length (dashed line).

Similar to the results in [26], at low pump intensities the Raman laser mode size in the plane-plane cavity tends to infinity, while that of the micro-lensed cavity is determined by the radius of curvature of the input mirror and the length of the Raman medium. After a certain pump intensity $\left(\sim 0.1 \mathrm{GW} / \mathrm{cm}^{2}\right)$ the mode sizes in both resonators become equal. The resonator mode radii at pump intensities above this level are dictated by the Raman gain rather than the $\mathrm{RoC}$ of the resonator mirrors (in the case of the micro-lensed device) and cavity lengths [24-26]. In this regime, the resonator mode radius $\omega_{D}$ can be calculated as [25]:

$$
\omega_{D}=\sqrt{\omega_{P} \sqrt{\frac{\lambda_{s}}{2 \pi g I_{P} n}}} \text { (7). }
$$

From the above equation it can be seen that Raman gainguiding tends to reduce the radius of the intracavity beam with higher pump intensity. This trend is evident, for both the planeplane and micro-lensed cavities, in Fig. 6.

In the case of $2^{\text {nd }}$ Stokes emission, the intracavity $1^{\text {st }}$ Stokes field acts as the pump source for the $2^{\text {nd }}$ Stokes. Once the $2^{\text {nd }}$ Stokes reaches its threshold, the intensity of the $1^{\text {st }}$ Stokes stays constant at the threshold level [27]. This threshold intensity of the $2^{\text {nd }}$ Stokes oscillation will be about two times higher in the $1 \mathrm{~mm}$ long device in comparison with the $2 \mathrm{~mm}$ long one. Therefore, with the Raman gain-guiding effect one would expect the intracavity mode size of the $2^{\text {nd }}$ Stokes beam in the 1 $\mathrm{mm}$ long device to be smaller than the one in the $2 \mathrm{~mm}$ long device, which is what was experimentally observed here: the measured beam radius was $10.5-10.8 \mu \mathrm{m}$ in the $1 \mathrm{~mm}$ long 
device and $12.7-14 \mu \mathrm{m}$ in the $2 \mathrm{~mm}$ long one (Fig. 5).

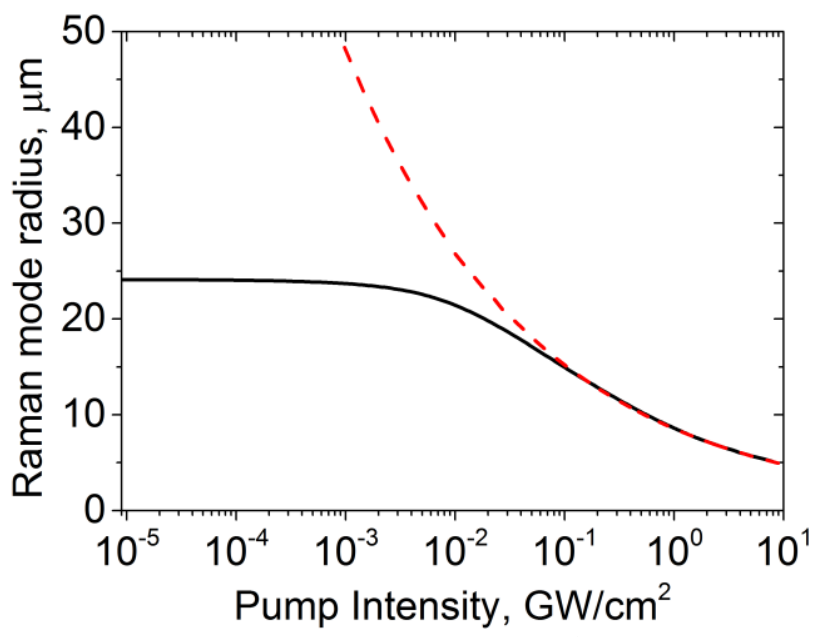

Fig. 6. The calculated resonator mode size of the $1^{\text {st }}$ Stokes emission on the output mirror as a function of pump intensity in a micro-lensed (solid line) and plane-plane (dashed line) devices with a length of $2 \mathrm{~mm}$.

We can now calculate the intracavity $2^{\text {nd }}$ Stokes beam size on the plane output mirror of the micro-lensed devices and compare the calculations with the experimental results presented in Fig. 5. Prior to performing the calculation the following considerations should be taken into account.

First, the pump source for the $2^{\text {nd }}$ Stokes emission is the $1^{\text {st }}$ Stokes field, therefore to calculate of the radius of the Gaussian duct of the $2^{\text {nd }}$ Stokes emission, one needs to know the radius of the $1^{\text {st }}$ Stokes beam. Therefore eq. (7) can be first used for calculation of the Gaussian duct of the $1^{\text {st }}$ Stokes oscillation.

Second, we should take into account that the Raman gain $g$ for a focused pump beam is calculated as [24, 28, 29]:

$$
g=\frac{4 g_{p}}{\lambda_{p}+\lambda_{s}} \tan ^{-1}\left(\frac{L}{b}\right) \frac{A}{L}
$$

where $g_{p}$ is the plane-wave Raman gain coefficient, $\lambda_{p}$ is the pump wavelength, $\lambda_{\mathrm{s}}$ is the Stokes wavelength, $A$ is the pump area and $b$ is the pump confocal parameter.

Finally, the pump intensity $I_{P}$ for calculation of the Gaussian duct of the $2^{\text {nd }}$ Stokes emission is the intracavity intensity of the $1^{\text {st }}$ Stokes required to achieve the threshold of the $2^{\text {nd }}$ Stokes oscillation. This threshold intensity, similarly to a conventional laser [21], can be calculated as:

$$
I_{t h}=\frac{-\ln R+2 \alpha L}{2 g_{573} L}(8),
$$

where $R$ is the output mirror reflectivity at the $2^{\text {nd }}$ Stokes wavelength (50\% at $620 \mathrm{~nm}), \alpha$ is the absorption coefficient at the $2^{\text {nd }}$ Stokes wavelength $\left(\sim 0.05 \mathrm{~cm}^{-1}\right.$ at $\left.620 \mathrm{~nm}\right)$ and $g_{573}$ is the Raman gain for a focused beam at the $1^{\text {st }}$ Stokes wavelength $(573 \mathrm{~nm})$, which in this case acts as the pump for the $2^{\text {nd }}$ Stokes emission and can be calculated using eq. (9).

With all these considerations taken into account, eq. (7) for the $1^{\text {st }}\left(\omega_{1}\right)$ and $2^{\text {nd }}$ Stokes $\left(\omega_{2}\right)$ Gaussian ducts radii can be rewritten as:
TABLE I

PARAMETERS FOR GAUSSIAN DUCT CALCULATION

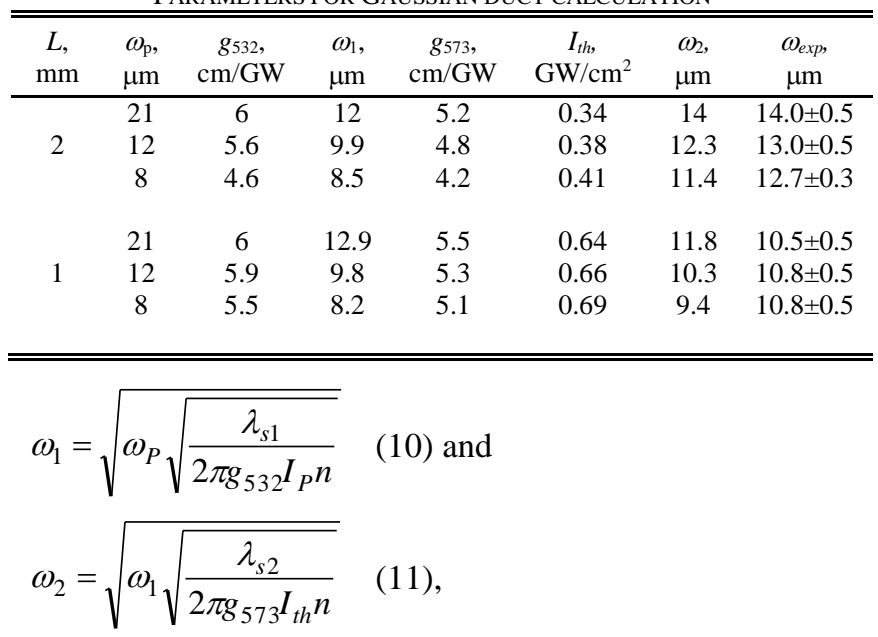

where $\lambda_{s l}$ is the $1^{\text {st }}$ Stokes wavelength, $\lambda_{s 2}$ is the $2^{\text {nd }}$ Stokes wavelength and $g_{532}$ is, similar to $g_{573}$, the Raman gain for a focused beam at the pump wavelength of $532 \mathrm{~nm}$ calculated using eq. (9).

The parameters used for calculations are summarized in Table I, along with the experimental values of the $2^{\text {nd }}$ Stokes intracavity mode radius $\omega_{\text {exp }}$ on the output mirror of each of the micro-lensed devices. The plane-wave Raman gain coefficients $g_{p}$ at 532 and $573 \mathrm{~nm}$, which were used to calculate $g_{532}$ and $g_{573}$, are taken as 30 and $28 \mathrm{~cm} / \mathrm{GW}$ correspondingly [30] for pump polarization along $\langle 100\rangle$ (33\% lower than the values obtained in [30] for polarization along the <111> axis of diamond). The plane-wave Raman gain coefficient at $573 \mathrm{~nm}$ was not directly measured in [30], rather it is calculated from the value measured at $532 \mathrm{~nm}$ taking into account that the Raman gain scales linearly with the pump wavenumber [30].

The calculated radii of the Gaussian duct of the $2^{\text {nd }}$ Stokes emission in the micro-lensed devices, $\omega_{2}$, are 14, 12.3 and 11.4 $\mu \mathrm{m}$ for the $2 \mathrm{~mm}$ long device and 11.8, 10.3 and $9.4 \mu \mathrm{m}$ for the $1 \mathrm{~mm}$ long device, for the different pump spot radii $\omega_{\mathrm{p}}(21,12$ and $8 \mu \mathrm{m}$ ) used in the experimental work (Table I). The obtained values are in good agreement with the experimentally measured $2^{\text {nd }}$ Stokes intracavity mode radii $\omega_{\text {exp }}$ (Fig. 5 and Table I). This suggests that Raman gain-guiding is a plausible mechanism responsible for beam formation in Raman lasers.

\section{Raman gain-guiding and thermal lens in Raman lasers}

The estimates at the end of the Section II indicate that at the power levels we used in this work there are not any significant thermal effects expected in our devices. Therefore, thermal lensing is unlikely to be the reason that the measured $2^{\text {nd }}$ Stokes beam size on the output mirror of our micro-lensed devices is smaller than the one expected from the ABCD analysis of the Raman resonator. However, if higher powers are used both gain-guiding and thermal effects will be present. Here we briefly consider the effect the competition between thermal lensing and Raman gain-guiding has on stability of the Raman resonator.

In Raman lasers, the heat load caused by Raman oscillation 
may compete with the Raman gain-guiding effect and after a certain power threshold the Raman laser cavity might become unstable. This is similar to the effect of the heat load on the refractive index guiding in fibre lasers [31]. The critical Stokes power $P_{\text {St,cr }}$ which creates a thermal lens with sufficient optical power to compete with the Raman gain-guiding effect is [22, 31]:

$P_{S t, c r}=\frac{2}{\pi n} \frac{k \lambda_{s}^{2}}{d n / d T \omega^{2}} \frac{L}{\lambda_{s} / \lambda_{P}-1}$ (12),

where $k$ is the thermal conductivity, $\lambda_{\mathrm{P}}$ is the pump wavelength, $\lambda_{\mathrm{s}}$ is the Stokes wavelength, $\mathrm{n}$ is the refractive index, $d n / d T$ is the thermo-optic coefficient, $L$ is the sample length and $\omega$ is the radius of the Gaussian duct of the Stokes emission.

For example, by pumping diamond at $\lambda_{\mathrm{P}}=1064 \mathrm{~nm}$ (giving a $1^{\text {st }}$ Stokes wavelength of $\lambda s=1240 \mathrm{~nm}$ ) and assuming the radius of the Gaussian duct is $20 \mu \mathrm{m}$ in an $8 \mathrm{~mm}$ long diamond crystal, the expected critical Stokes power would be $10 \mathrm{~kW}$ (in diamond $k=2000 \mathrm{Wm}^{-1} \mathrm{~K}^{-1}$ and $\left.d n / d T=9.6 \times 10^{-6} \mathrm{~K}^{-1}[32]\right)$.

This calculation indicates that Raman output power from the monolithic micro-lensed devices can reach significant values without any issues related to the thermal lensing effects which would influence the stability of the Raman resonator. For example, this may explain the reason why the expected optical power of the thermal lens in an $8 \mathrm{~mm}$ long diamond did not have any effect on the performance and stability of the $381 \mathrm{~W}$ CW diamond Raman laser realized in [33].

\section{CONCLUSION}

Energy and wavelength scalability of monolithic diamond Raman lasers has been demonstrated. Under $532 \mathrm{~nm}$ pumping, 1st Stokes laser emission at $573 \mathrm{~nm}$ (yellow) with a pulse energy of $3.1 \mathrm{~mJ}$ was achieved in a plane-plane monolithic diamond Raman laser with a conversion efficiency of $40 \%$. This represents a 300-fold increase in pulse energy in comparison with our previous work on monolithic DRLs. The flexibility of the monolithic DRL technology for extending the spectral coverage of commercial lasers emitting in the green, was also demonstrated: 2nd Stokes oscillation at $620 \mathrm{~nm}$ from a monolithic micro-lensed converter was reported with the highest pump to 2nd Stokes conversion efficiency being $63 \%$ in a $2 \mathrm{~mm}$ long device. The $2^{\text {nd }}$ Stokes pulse duration was $1 \mathrm{~ns}$ for $1.5 \mathrm{~ns}$ pump pulse duration and the best Raman beam $\mathbf{M}^{2}$ was measured to be $2.0 \times 1.7$. The slope efficiency of the $2^{\text {nd }}$ Stokes power was found to be higher for tighter pump focusing.

The intracavity $2^{\text {nd }}$ Stokes Raman laser mode radius was experimentally measured for different pump focusing conditions in 2- and $1 \mathrm{~mm}$ long micro-lensed monolithic diamond Raman laser devices. It was found to be $\sim 2$ times smaller than the one expected from the ABCD matrix calculations of the resonator mode. This intracavity $2^{\text {nd }}$ Stokes laser mode radius was re-calculated based on the assumption of a Raman gain-guiding mechanism contributing to the laser mode formation in monolithic diamond Raman lasers. We found good agreement between the measured values of the intracavity Raman mode sizes and the ones calculated under the assumption of Raman gain-guiding. If this Raman gain-guiding effect is taken into account, our calculations indicate that the critical Stokes power at which the thermal lens destabilizes the Raman laser mode may be significantly higher than what would otherwise be expected, at least in CW Raman lasers. This suggests that the Raman gain-guiding may play a significant role in determining the power scaling potential of these devices. The effect of the heat capacity on the dynamic thermal lens in high energy pulsed Raman laser should be studied in more detail, and further clarification of the exact role of Raman gainguiding may therefore be crucial for the successful development of high power CW and high energy pulsed Raman lasers.

\section{ACKNOWLEDGMENT}

Keith Oakes from Elforlight is acknowledged for FQS laser loan.

\section{REFERENCES}

[1] M. Xu and L. V. Wang, "Photoacoustic imaging in biomedicine," Review of Scientific Instruments, vol. 77, p. 041101, 2006.

[2] L. V. Wang and S. Hu, "Photoacoustic Tomography: In Vivo Imaging from Organelles to Organs," Science, vol. 335, pp. 1458-1462, 2012.

[3] S. Hu and L. V. Wang, "Photoacoustic imaging and characterization of the microvasculature," Journal of Biomedical Optics, vol. 15, p. 011101, 2010.

[4] J. M. Yarborough, "cw dye laser emission spanning the visible spectrum," Applied Physics Letters, vol. 24, pp. 629-630, 1974.

[5] R. Reisfeld, D. Brusilovsky, M. Eyal, E. Miron, Z. Burstein, and J. Ivri, "A new solid-state tunable laser in the visible," Chemical Physics Letters, vol. 160, pp. 43-44, 1989/07/28/ 1989.

[6] R. M. Gerosa, A. Sudirman, L. d. S. Menezes, W. Margulis, and C. J. S. de Matos, "All-fiber high repetition rate microfluidic dye laser," Optica, vol. 2, pp. 186-193, 2015/02/20 2015.

[7] Y. X. Fan, R. C. Eckardt, R. L. Byer, J. Nolting, and R. Wallenstein, "Visible $\mathrm{BaB}_{2} \mathrm{O}_{4}$ optical parametric oscillator pumped at $355 \mathrm{~nm}$ by a single-axial-mode pulsed source," Applied Physics Letters, vol. 53, pp. 2014-2016, 1988.

[8] M. Ebrahimzadeh, M. H. Dunn, and F. Akerboom, "Highly efficient visible urea optical parametric oscillator pumped by a $\mathrm{XeCl}$ excimer laser," Optics Letters, vol. 14, pp. 560-562, 1989/06/01 1989.

[9] J. Sperling and K. Hens, "Made Easy: CW Laser Light Widely Tunable Across the Visible," Optik \& Photonik, vol. 13, pp. 22-24, 2018.

[10] P. Črný, H. Jelínková, P. G. Zverev, and T. T. Basiev, "Solid state lasers with Raman frequency conversion," Progress in Quantum Electronics, vol. 28, pp. 113-143, 2004/01/01/2004.

[11] A. I. Vodchits, D. N. Busko, V. A. Orlovich, V. A. Lisinetskii, A. S. Grabtchikov, P. A. Apanasevich, et al., "Multi-frequency quasicontinuous wave solid-state Raman laser for the ultraviolet, visible, and near infrared," Optics Communications, vol. 272, pp. 467-475, 2007/04/15/ 2007

[12] H. M. Pask, P. Dekker, R. P. Mildren, D. J. Spence, and J. A. Piper, "Wavelength-versatile visible and UV sources based on crystalline Raman lasers," Progress in Quantum Electronics, vol. 32, pp. 121-158, 2008/01/01/ 2008.

[13] A. J. Lee, D. J. Spence, J. A. Piper, and H. M. Pask, "A wavelengthversatile, continuous-wave, self-Raman solid-state laser operating in the visible," Optics Express, vol. 18, pp. 20013-20018, 2010.

[14] A. Kananovich, A. Demidovich, M. Danailov, A. Grabtchikov, and V. Orlovich, "All-solid-state quasi-CW yellow laser with intracavity selfRaman conversion and sum frequency generation," Laser Physics Letters, vol. 7, pp. 573-578, 2010.

[15] D. C. Parrotta, A. J. Kemp, M. D. Dawson, and J. E. Hastie, "Multiwatt, Continuous-Wave, Tunable Diamond Raman Laser With Intracavity Frequency-Doubling to the Visible Region," IEEE Journal of Selected Topics in Quantum Electronics, vol. 19, pp. 1400108-1400108, 2013.

[16] S. Reilly, V. G. Savitski, H. Liu, E. Gu, M. D. Dawson, and A. J. Kemp, "Monolithic diamond Raman laser," Optics Letters, vol. 40, pp. 930-933, 2015. 
[17] J. Nikkinen, A. Härkönen, and M. Guina, "Sub-50 ps pulses at $620 \mathrm{~nm}$ obtained from frequency doubled $1240 \mathrm{~nm}$ diamond Raman laser," Optics Express, vol. 25, pp. 30365-30370, 2017/11/27 2017.

[18] J. Nikkinen, V. Savitski, S. Reilly, Ł. Dziechciarczyk, A. Härkönen, A. Kemp, et al., "Sub-100 ps monolithic diamond Raman laser emitting at 573 nm," IEEE Photonics Technology Letters, vol. PP, pp. 1-1, 2018.

[19] H. Liu, S. Reilly, J. Herrnsdorf, E. Xie, V. G. Savitski, A. J. Kemp, et al., "Large radius of curvature micro-lenses on single crystal diamond for application in monolithic diamond Raman lasers," Diamond and Related Materials, vol. 65, pp. 37-41, 5// 2016.

[20] R. P. Mildren, J. E. Butler, and J. R. Rabeau, "CVD-diamond external cavity Raman laser at 573 nm," Optics Express, vol. 16, pp. 18950-18955, 2008.

[21] A. E. Siegman, Lasers: University Science Books, 1986.

[22] H. M. Pask, "The design and operation of solid-state Raman lasers," Progress in Quantum Electronics, vol. 27, pp. 3-56, 2003.

[23] V. P. Pashinin, V. G. Ralchenko, A. P. Bolshakov, E. E. Ashkinazi, M. A. Gorbashova, V. Y. Yurov, et al., "External-cavity diamond Raman laser performance at $1240 \mathrm{~nm}$ and $1485 \mathrm{~nm}$ wavelengths with high pulse energy," Laser Physics Letters, vol. 13, p. 065001, 2016.

[24] D. Cotter, D. C. Hanna, and R. Wyatt, "Infrared stimulated Raman generation: Effects of gain focussing on threshold and tuning behaviour," Applied physics, vol. 8, pp. 333-340, December 011975.

[25] H. Kogelnik, "On the propagation of Gaussian beams of light through lenslike media including those with a loss or gain variation," Applied Optics, vol. 4, pp. 1562-1569, 1965.

[26] L. Casperson and A. Yariv, "The Gaussian mode in optical resonators with a radial gain profile," Applied Physics Letters, vol. 12, pp. 355-357, 1968.

[27] A. Penzkofer, A. Laubereau, and W. Kaiser, "High-Intensity Raman Interactions," Progress in Quantum Electronics, vol. 6, pp. 56-140, 1979.

[28] A. Glass, "7.4 - Design considerations for Raman lasers," IEEE Journal of Quantum Electronics, vol. 3, pp. 516-520, 1967.

[29] W. R. Trutna and R. L. Byer, "Multiple-pass Raman gain cell," Applied Optics, vol. 19, pp. 301-312, 1980/01/15 1980.

[30] V. G. Savitski, S. Reilly, and A. J. Kemp, "Steady-State Raman Gain in Diamond as a Function of Pump Wavelength," IEEE J. Quantum Electron., vol. 49, pp. 218-223, 2013.

[31] J. W. Dawson, M. J. Messerly, R. J. Beach, M. Y. Shverdin, E. A. Stappaerts, A. K. Sridharan, et al., "Analysis of the scalability of diffraction-limited fiber lasers and amplifiers to high average power," Optics Express, vol. 16, pp. 13240-13266, 2008/08/18 2008.

[32] I. Friel, S. L. Geoghegan, D. J. Twitchen, and G. A. Scarsbrook, "Development of high quality single crystal diamond for novel laser applications," in Optics and Photonics for Counterterrorism and Crime Fighting VI and Optical Materials in Defence Systems Technology VII, Toulouse, France, 2010, pp. 783819-8.

[33] R. J. Williams, J. Nold, M. Strecker, O. Kitzler, A. McKay, T. Schreiber, et al., "Efficient Raman frequency conversion of high-power fiber lasers in diamond," Laser \& Photonics Reviews, vol. 9, pp. 405-411, 2015.

Vasili G. Savitski received the Engineering Diploma in optics from the Belarus National Technical University, Minsk, in 1999, and the Ph.D. degree from the Institute of Physics, Minsk, in 2005 for work on the nonlinear optics of quantum dots in glasses. He worked at the International Laser Center, Minsk on the nonlinear optics of quantum dots, passive mode locking and thermal effects in lasers from 1999 to 2007. Currently, he is with the Institute of Photonics, University of Strathclyde, Glasgow.

Giorgos Demetriou received the Diploma in electrical engineering and computer science from the National Technical University of Athens, Athens, Greece, in 2012. He received the Ph.D. degree in physics at Heriot Watt University, Edinburgh, U.K in 2016. Short after receiving his Ph.D. he joined the Institute of Photonics, University of Strathclyde, Glasgow, where he works as a research associate on solid state laser engineering. Dr Demetriou is a member of the Optical Society of America.

Sean Reilly received the M.Sci. degree in physics from the University of Strathclyde, U.K., in 2011, and the Ph.D. degree from the University of Strathclyde in 2015.

Hangyu Liu received the B. Eng. from the Wuhan University of Technology, China, in 2012, and the Ph.D. degree from the University of Strathclyde in 2016.
Erdan Gu has been working in the Institute of Photonics, University of Strathclyde, Glasgow since July, 2002. He is an Associate Director and a Research Team Leader at the Institute. He obtained his $\mathrm{PhD}$ degree on thin film physics from Aberdeen University, UK in 1992. Then, he worked as a research fellow in Cavendish Laboratory, Cambridge University, UK. In 1997, he joined the thin film group, Oxford Instruments plc, UK as a senior research scientist working on superconducting photonic devices. In the Institute of Photonics, he is working and playing a leading role in a range of research projects on photonic materials and devices, micro/nano optoelectronics, diamond photonics, optoelectronic devices for visible light communications.

Martin D. Dawson (M'85-SM'98-F'09) received the PhD degree in physics from Imperial College, London, in 1985. He has worked at North Texas State University, Denton, the University of Iowa, Iowa City, Sharp Laboratories of Europe, Oxford and the University of Strathclyde, where he has been a professor since 2001. He is Director of Research at the University of Strathclyde's Institute of Photonics and Head of the Fraunhofer Centre for Applied Photonics.

Alan J. Kemp (M'07) received the B.Sc. degree (Hons.) from the University of Glasgow, Glasgow, U.K., in 1996, and the Ph.D. degree from the University of St. Andrews, St. Andrews, U.K., in 1999, for work on microchip lasers. He worked on ultrafast lasers with the University of St. Andrews from 1999 to 2002. In 2002, he moved to the Institute of Photonics, University of Strathclyde, Glasgow, where he works on solid-state laser engineering, and holds a Fraunhofer UK / Royal Academy of Engineering Research Chair (2014-19). 